\title{
Distribución geográfica del género Gyriosomus Guérin-Méneville, 1834 (Coleoptera: Tenebrionidae): una aproximación biogeográfica
}

\author{
Geographical distribution of the genus Gyriosomus Guérin-Méneville, 1834 \\ (Coleoptera: Tenebrionidae): a biogeographic approach
}

\author{
JAIME PIZARRO-ARAYA ${ }^{1,2} \&$ VIVIANE JEREZ ${ }^{3}$ \\ ${ }^{1}$ Departamento de Zoología, Facultad de Ciencias Naturales y Oceanográficas, Universidad de Concepción, Casilla 160-C, \\ Concepción, Chile; \\ e-mail: jaimepizarro@udec.cl \\ ${ }^{2}$ Dirección actual: Laboratorio de Entomología Ecológica, Departamento de Biología, Facultad de Ciencias, \\ Universidad de La Serena, Casilla 599, La Serena, Chile \\ ${ }^{3}$ Departamento de Zoología, Facultad de Ciencias Naturales y Oceanográficas, \\ Universidad de Concepción, Casilla 160-C, Concepción, Chile; \\ e-mail: vijerez@udec.cl
}

\begin{abstract}
RESUMEN
Se realizó un análisis de parsimonia de endemismo para el género Gyriosomus, taxón monofilético, erémico y endémico del norte de Chile. En este trabajo se evalúa (i) si los patrones de distribución de las especies de Gyriosomus están determinados por la heterogeneidad del hábitat y (ii) si las discontinuidades distribucionales de los taxa reflejan áreas de endemismo. Para ello se determinaron áreas de endemismo para las especies de Gyriosomus, se relacionaron con formaciones vegetacionales, y se establecieron las relaciones biogeográficas entre los distintos ecosistemas descritos para el norte de Chile. El área de estudio correspondió a la distribución del género y fue dividida en cuadrículas de un cuarto de grado de latitud y medio grado de longitud; los datos de presencia o ausencia de las especies se registraron en una matriz, la que se analizó mediante los programas NONA 2.0 y Winclada. Un árbol de consenso estricto permitió establecer para Gyriosomus, un patrón de áreas anidadas, donde se reconocieron dos áreas de endemismo en los ecosistemas costeros de Paposo y Carrizal Bajo. El desierto costero de Taltal fue determinado por G. angustus y G. curtisi y el desierto costero de Huasco con $G$. planatus y G. kingi. Los ecosistemas de desierto costero de Huasco y matorral estepario costero presentaron la mayor riqueza de especies de Gyriosomus. Un cladograma reveló una divergencia en el componente faunístico en dos unidades biogeográficas, el desierto costero y matorral estepario. Los resultados de este trabajo complementados con una reconstrucción filogenética del género podrían ser utilizados para entender los eventos vicariantes que explicarían las divergencias evolutivas de Gyriosomus.
\end{abstract}

Palabras clave: biogeografía, Tenebrionidae, Gyriosomus, áreas de endemismo, desiertos costeros.

\begin{abstract}
A parsimony analysis of areas of endemism for the monophyletic genus Gyriosomus, an eremic and endemic element of the northern coast of Chile, was performed. We tested that: (i) distributional patterns of Gyriosomus species are determined by environmental heterogeneity, and that (ii) discontinuities in the distribution of Gyriosomus species will reflect areas of endemism. We identified areas of endemism for Gyriosomus species, relating them with vegetational formations, and establishing biogeographical patterns among different ecosystems from northern Chile. The study area corresponded with the distributional limits of the genus from where data on the presence or absence of 34 species in grid cells half degree longitude by one quarter degree were registered on a matrix. The analysis was undertaken using NONA 2.0 and Winclada softwares. A strict consensus tree allowed us to establish a pattern of nested areas for Gyriosomus, where two areas of endemism were recognized; coastal ecosystems of Paposo and Carrizal Bajo. Taltal coastal desert was determined for G. angustus and G. curtisi and the Huasco coastal desert with G. planatus and G. kingi. The Huasco coastal desert followed by the coastal steppe shrub ecosystems were the richest areas in terms of Gyriosomus species. A cladogram revealed disjunction of faunistic components in two biogeographic units: the coastal desert and the steppe shrub, although several species are widely distributed along the intermediate depression. The results of this study along with a phylogenetic reconstruction of the genus might explain vicariant events behind the biogeographic disjunction of Gyriosomus.
\end{abstract}

Key words: biogeography, Tenebrionidae, Gyriosomus, areas of endemism, coastal desert. 


\section{INTRODUCCIÓN}

El conocimiento detallado de la distribución de la diversidad biológica, permite su interpretación biogeográfica identificando áreas naturales que concentren por una parte la mayor cantidad de taxa endémicos y, por otra, que estos endemismos reflejen la historia evolutiva de la diversidad y su relación con biotas ancestrales. Asimismo, los análisis biogeográficos pueden ser complementados con índices de diversidad filogenética y de riqueza de especies presentes en dichas áreas (Eguiarte et al. 1999, Camus 2001).

Entre los métodos utilizados por la biogeografía histórica, el análisis de parsimonia de endemismos (Parsimony Analysis of Endemicity = PAE) (Morrone 1994, Escalante \& Morrone 2003) permite clasificar áreas o localidades de acuerdo a taxa compartidos (Morrone \& Crisci 1995) y al mismo tiempo, detectar áreas con concentración de especies endémicas (Posadas 1996, Posadas \& Miranda-Esquivel 1999, Cavieres et al. 2001, 2002).

Los taxa endémicos son aquellos que están delimitados a algún lugar geográfico específico y pueden considerarse como la diversidad biológica única de un lugar, sensu Cavieres et al. (2001). Por el hecho de tener un rango de distribución restringido, los taxa endémicos tienen mayores probabilidades de extinguirse (Myers et al. 2000), por lo cual la delimitación de áreas de endemismo es fundamental para formular estrategias dirigidas al uso sustentable y la conservación de la biodiversidad (Amat-García \& Miranda-Esquivel 1996, Szumik et al. 2002).

La mayoría de los estudios biogeográficos realizados para insectos de Sudamérica han estado dirigidos a comparar la similitud biótica entre la región Neotropical y distintas áreas del mundo, basándose en taxones compartidos con los restantes continentes australes (Kuschel 1964, 1969, Morrone et al. 1994). Autores como Peña (1966a), Morrone et al. (1997), Roig-Juñent \& Flores (2001), Morrone et al. (2002), Donato et al. (2003), han realizado estudios, utilizando taxones propios de Sudamérica austral, principalmente Coleoptera, mientras que Morrone (1996) y Roig-Juñent et al. (2003) realizaron análisis biogeográficos utilizando artrópodos epígeos.

El conocimiento actual de áreas de endemismo en Chile se reduce a los aportes de Posadas (1996) para la XII Región, Squeo et al. (1998) y Cavieres et al. (2002) para la II Región, y Cavieres et al. (2001) para la IV Región de Chile, quienes establecen para la vegetación áreas de concentración de especies endémicas; en términos faunísticos, solamente Jerez (2000) delimita áreas de endemismo para la II Región en base a la fauna de Coleópteros.

Las franjas longitudinales costeras que existen en el norte de Chile, corresponden a distintas zonas ecológicas y geomorfológicas (Paskoff 1993), con una elevada heterogeneidad de hábitats que han permitido la adaptación y evolución de los organismos (Ormazábal 1993, Gajardo 1994), originando un alto grado de diversidad y endemismo en su biota (Cabrera \& Willink 1973).

En estos ecosistemas, numerosos géneros de insectos han experimentado radiaciones evolutivas significativas como es el caso de Gyriosomus, uno de los géneros más diversificados de Nycteliini, tribu endémica de ambientes áridos y semiáridos de Sudamérica austral (Flores 2000). Gyriosomus, taxón monofilético con 34 especies descritas (Flores 1997) es un importante elemento erémico y endémico de la biota del norte costero de Chile (Peña 1959, RoigJuñent \& Flores 2001) y la mayoría de sus especies presentan irrupciones poblacionales relacionadas con ocurrencias de El Niño Oscilación del Sur (ENOS) (Cepeda-Pizarro 1989, Pizarro et al. $\left.2001^{1}\right)$.

Este género se distribuye entre los $25^{\circ}$ y $34^{\circ}$ $\mathrm{S}$ y sus especies están asociadas a la vegetación arbustiva y herbácea (perennes y anuales) de terrazas costeras, planicies y cuencas de la Depresión intermedia.

Dada la heterogeneidad de ambientes característicos de la región mediterránea perárida, semiárida y árida de Chile (Di Castri 1968), cabría esperar que las especies de Gyriosomus, mostraran patrones distribucionales concordantes con los diferentes ecosistemas descritos en el área y que las discontinuidades distribucionales presentadas por las especies se reflejaran en áreas de endemismo.

Los objetivos del presente trabajo fueron por lo tanto, determinar áreas de endemismo para las especies de Gyriosomus mediante un análisis de parsimonia de endemismo, relacionar estas áreas con formaciones vegetacionales, e interpretar las relaciones biogeográficas que puedan existir entre los distintos ecosistemas descritos para el norte de Chile.

\footnotetext{
${ }^{1}$ PIZARRO J, S VEGA \& J CEPEDA (2001) Efecto del mes de muestreo y del tipo de hábitat sobre la denso-actividad de la Tenebrionidofauna epígea en un ecosistema desértico costero de Chile. XXIII Congreso Nacional de Entomología, Temuco, Chile, Libro de Resúmenes: 6.
} 


\section{MATERIALES Y MÉTODOS}

\section{Distribución geográfica}

La distribución geográfica de Gyriosomus se actualizó a partir de la revisión de material de referencia conservado en las colecciones entomológicas del Museo de Zoología de la Universidad de Concepción (MZUC), Museo Nacional de Historia Natural, Santiago (MNHN), Instituto de Entomología, Universidad Metropolitana de Ciencias de la Educación, Santiago (IEUM$\mathrm{CE})$, Laboratorio de Entomología Ecológica del Departamento de Biología de la Universidad de La Serena, La Serena (LEULS), Laboratorio de Entomología, del Instituto Argentino de Investigaciones de las Zonas Áridas (IADIZA), Argentina y colección particular de Juan Enrique Barriga (JEBC).

La información se complementó con datos distribucionales señalados en literatura, en particular los trabajos de: Waterhouse (1844), Fairmaire (1876), Philippi (1887), Gebien (1910), Kulzer (1954, 1959), Peña (1966a,b) y Kaszab (1969), además con prospecciones realizadas entre septiembre y noviembre de 2002 y abril de 2003, abarcando parte de la II, III y IV Región (Tabla 1). Todas las localidades de muestreo fueron referenciadas geográficamente mediante GPS (Etrex-Personal Navigator, Gamin) y el material recolectado se identificó a nivel específico mediante literatura especializada y comparación con material de museo. El material recolectado está depositado en las colecciones del Museo de Zoología de la Universidad de Concepción y del Laboratorio de Entomología Ecológica de la Universidad de La Serena.

\section{Análisis de parsimonia de endemismos}

El área de distribución del género entre $25^{\circ}$ y $34^{\circ} \mathrm{S}$, se dividió en cuadrículas de medio grado de longitud por un cuarto de latitud (Fig. 1), registrando la presencia de 34 especies y eliminando aquellas cuadrículas para las que no existe información. Para la delimitación de áreas de endemismo se aplicó el PAE, siguiendo la metodología utilizada por Morrone (1994), Morrone et al. (1997), Posadas \& Miranda-Esquivel (1999), Jerez (2000), Cavieres et al. (2001, 2002), Fattorini (2002), García-Barros et al. (2002), Morrone \& Escalante (2002), Aguilar-Aguilar et al. (2003), Escalante \& Morrone (2003), Escalante et al. (2003), EspadasManrique et al. (2003). Para ello, se elaboró una matriz, donde las columnas representan las cuadrículas y las filas las especies; se agregó una fila hipotética con ausencias para enraizar los cladogramas y se registró la ausencia o presencia $(0,1)$ de cada especie en las diferentes cuadrículas. El análisis de la matriz se realizó con el programa NONA 2.0 (Goloboff 1997), por medio de búsquedas heurísticas, con los parámetros "hold 10.000, hold/10, mult*100, max*". El árbol de consenso estricto se calculó con el programa Winclada (Nixon 2000).

\section{Formaciones vegetacionales}

La distribución espacial de las especies de Gyriosomus se relacionó con formaciones vegetacionales descritas por Gajardo (1994) entre Paposo y Rancagua $\left(25^{\circ}-34^{\circ} \mathrm{S}\right)$ y que corresponden al desierto costero de Taltal, desierto costero del Huasco, desierto florido de los Llanos, desierto florido de las serranías, matorral estepario costero, matorral estepario boscoso, matorral estepario interior, matorral estepario arborescente, matorral andino esclerófilo, bosque esclerófilo costero y matorral espinoso de la cordillera de la costa (Tabla 2).

\section{RESULTADOS}

\section{Análisis de parsimonia de endemismos}

Se obtuvo 100 cladogramas igualmente parsimoniosos de 66 pasos, resumidos en un árbol de consenso estricto, a partir del cual se pudo determinar dos áreas de endemismo, cada una definida por la presencia de dos sinapomorfías: Paposo y Carrizal Bajo (Fig.1). Paposo se sustenta por la presencia de G. curtisi y G. angustus y Carrizal Bajo por G. planatus y G. kingi. En el cladograma se observa que algunas especies están presentes en cuadrículas únicas como ocurre con G. foveopunctatus en la cuadrícula $25, G$. modestus en la 36 y G. coriaceus en la 37. Además se determinó a G. laevigatus como especie sinapomórfica para un conjunto mayor de cuadrículas.

En relación con aspectos ecológicos, Paposo corresponde al sector costero de la quebrada de Paposo (Provincia de Antofagasta: II Región) y Carrizal Bajo está comprendido en el sector de planicies litorales de la Provincia de Huasco: III Región de Chile.

El número de especies de Gyriosomus presentes en cada formación vegetal se entrega en la Tabla 2. Se observa que la mayor riqueza específica se encuentra en sectores costeros como el desierto costero del Huasco (DCH) con 11 especies y Matorral estepario costero (MEC) con 12 especies. Dentro de los sistemas interio- 


\section{TABLA 1}

Listado de especies de Gyriosomus y ubicación en las cuadriculas según Fig. 1 Species listing of Gyriosomus, according to their location in square in Fig. 1

\begin{tabular}{|c|c|c|c|}
\hline ID & Especie & Cuadrícula & Fuente \\
\hline 1 & G. hoppei (Gray, 1832) & $20,21,22,24,26,29,32$ & $\begin{array}{l}\text { MNHN; IEUMCE; LEULS; IADIZA; Philippi, } \\
\text { 1887; Kulzer, 1959; Peña, 1966b; Kaszab, 1969; } \\
\text { JEBC. }\end{array}$ \\
\hline 2 & G. impressus Guerín-Méneville, 1834 & $13,15,17,19,22$ & $\begin{array}{l}\text { MNHN; LEULS; Kulzer, 1959; Peña, 1966b; } \\
\text { JEBC. }\end{array}$ \\
\hline 3 & G. laevigatus Guerín-Méneville, 1834 & $40,41,42,43,44,45,46$ & $\begin{array}{l}\text { MNHN; IEUMCE; Kulzer, 1959; Peña, 1966b; } \\
\text { Saíz et al., 1989; JEBC. }\end{array}$ \\
\hline 4 & G. luczotii Laporte, 1840 & $19,20,21,22,24$ & $\begin{array}{l}\text { MNHN; MZUC; IEUMCE; LEULS; IADIZA; Kul- } \\
\text { zer, 1959; Kaszab, 1969; JEBC. }\end{array}$ \\
\hline 5 & G. marmoratus Waterhouse, 1843 & $21,22,23,24,25$ & $\begin{array}{l}\text { MZUC; LEULS; IADIZA Kulzer, 1959; Peña, } \\
\text { 1966b. }\end{array}$ \\
\hline 6 & G. bridgesi Waterhouse, 1843 & $21,22,24,29,30$ & $\begin{array}{l}\text { MNHN; MZUC; IEUMCE; IADIZA; Philippi, } \\
\text { 1887; Kulzer, 1959; Peña, 1966b; Kaszab, 1969; } \\
\text { JEBC. }\end{array}$ \\
\hline 7 & G. elongatus Waterhouse, 1843 & 17,19 & $\begin{array}{l}\text { MNHN; IEUMCE; LEULS; IADIZA; Philippi, } \\
\text { 1887; Kulzer, 1959; Peña, 1966b; JEBC. }\end{array}$ \\
\hline 8 & G. whitei Waterhouse, 1844 & $11,13,17,18,19$ & $\begin{array}{l}\text { MNHN; LEULS; IADIZA; Waterhouse, 1844; } \\
\text { Kulzer, 1959; JEBC. }\end{array}$ \\
\hline 9 & G. planatus Solier, 1851 & $9,11,12,13$ & $\begin{array}{l}\text { MNHN; IEUMCE; LEULS; IADIZA; Solier, 1851; } \\
\text { Philippi, 1887; Kulzer, 1959; Peña, 1966b; JEBC. }\end{array}$ \\
\hline 10 & G. parvus Solier, 1851 & $11,12,13$ & $\begin{array}{l}\text { MNHN; MZUC; IADIZA; Solier, 1851; Philippi, } \\
\text { 1887; Kulzer, 1959; Peña, 1966b; JEBC. }\end{array}$ \\
\hline 11 & G. angustus Philippi, 1864 & 1 & $\begin{array}{l}\text { MNHN; LEULS; IADIZA; Kulzer, 1959; Peña, } \\
\text { 1966b. }\end{array}$ \\
\hline 12 & G. kingi Reed, 1873 & $9,11,12,13$ & $\begin{array}{l}\text { MNHN; IEUMCE; LEULS; IADIZA; Kulzer, } \\
\text { 1959; Peña, 1966b; JEBC. }\end{array}$ \\
\hline 13 & G. subrugatus Fairmare, 1876 & $11,13,16$ & $\begin{array}{l}\text { MNHN; MZUC; LEULS; Kulzer, 1959; Peña, } \\
\text { 1966b; Fairmare, 1876; JEBC. }\end{array}$ \\
\hline 14 & G. curtisi Fairmare, 1876 & 1 & $\begin{array}{l}\text { MNHN; IEUMCE; IADIZA; Fairmare, 1876; Kul- } \\
\text { zer, 1959; Peña, 1966b; JEBC. }\end{array}$ \\
\hline 15 & G. atacamensis Fairmare, 1876 & 11,13 & MNHN; Fairmare, 1876; Kulzer, 1959; Peña, 1966b \\
\hline 16 & G. foveopunctatus Fairmare, 1876 & 25 & $\begin{array}{l}\text { MNHN; Fairmare, 1876; Kulzer, 1959; Peña, } \\
\text { 1966b. }\end{array}$ \\
\hline 17 & G. batesi Fairmare, 1876 & $2,3,4,5,6,7,8,9,10,11,12,16$ & $\begin{array}{l}\text { MNHN; MZUC; IEUMCE; LEULS; IADIZA; } \\
\text { Fairmare, 1876; Kulzer, 1959; Peña, 1966b; JEBC }\end{array}$ \\
\hline 18 & G. paulseni Fairmare, 1886 & $33,34,35,36,38$ & $\begin{array}{l}\text { MNHN; MZUC; IEUMCE; Kulzer, 1959; Peña, } \\
\text { 1966b; JEBC. }\end{array}$ \\
\hline 19 & G. coriaceus Fairmare, 1886 & 37 & MNHN; Peña, 1966b. \\
\hline 20 & G. granocostatus Fairmare, 1886 & $32,33,36$ & MNHN; LEULS; Kulzer, 1959; Peña, 1966b; JEBC \\
\hline 21 & G. penicilliger Gebien, 1944 & $34,26,29$ & $\begin{array}{l}\text { MNHN; MZUC; LEULS; Kulzer, 1959; Peña, } \\
\text { 1966b; JEBC. }\end{array}$ \\
\hline 22 & G. planicollis Gebien, 1944 & $5,7,8,9,10,11,12$ & $\begin{array}{l}\text { MNHN; MZUC; IEUMCE; LEULS; IADIZA; Kul- } \\
\text { zer, 1959; Peña, 1966b; Gebien, 1944; JEBC. }\end{array}$ \\
\hline 23 & G. freyi Gebien, 1944 & $20,26,29,31,32,37$ & $\begin{array}{l}\text { MNHN; LEULS; IADIZA; Kulzer, 1959; Peña, } \\
\text { 1966b; Kaszab, 1969; Gebien, 1944; JEBC. }\end{array}$ \\
\hline 24 & G. leechi Kulzer, 1954 & $16,20,22,25,27,28,29,31,32$ & $\begin{array}{l}\text { MNHN; MZUC; IEUMCE; LEULS; IADIZA; Kul- } \\
\text { zer, 1954; Kaszab, 1969; Peña, 1966b; JEBC. }\end{array}$ \\
\hline 25 & G. reedi Kulzer, 1954 & $24,29,30,31,32,33,36$ & $\begin{array}{l}\text { MNHN; MZUC; LEULS; IADIZA; Kulzer, 1954; } \\
\text { Kaszab, 1969; Peña, 1966b; JEBC. }\end{array}$ \\
\hline 26 & G. amabilis Kulzer, 1959 & 19,20 & $\begin{array}{l}\text { MNHN; MZUC; IEUMCE; LEULS; IADIZA; } \\
\text { Peña, 1966b; JEBC. }\end{array}$ \\
\hline 27 & G. barriai Kulzer, 1959 & 19 & $\begin{array}{l}\text { MNHN; LEULS; IADIZA; Kulzer, 1959; Peña, } \\
\text { 1966b; JEBC. }\end{array}$ \\
\hline 28 & G. gebieni Kulzer, 1959 & $13,17,18$ & $\begin{array}{l}\text { MNHN; LEULS; IADIZA; Kulzer, 1959; Peña, } \\
\text { 1966b; JEBC. }\end{array}$ \\
\hline 29 & G. lucens Kulzer, 1959 & 36,38 & MNHN; Kulzer, 1959. \\
\hline 30 & G. pumilus Kulzer, 1959 & 32,33 & $\begin{array}{l}\text { MNHN; MZUC; LEULS; IADIZA; Kulzer, 1959, } \\
\text { Peña, 1966b. }\end{array}$ \\
\hline 31 & G. penai Kulzer, 1959 & 17,19 & $\begin{array}{l}\text { MNHN; MZUC; LEULS; Kulzer 1959; Peña, } \\
\text { 1966b. }\end{array}$ \\
\hline 32 & G. melcheri Kulzer, 1959 & $34,36,37$ & MZUC; LEULS; Kulzer, 1959; Peña, 1966b; JEBC. \\
\hline 33 & G. modestus Kulzer, 1959 & 36 & MZUC; Kulzer, 1959; Peña, 1966b; JEBC. \\
\hline 34 & G. kulzeri Peña, 1975 & 13 & Peña, 1975. \\
\hline
\end{tabular}




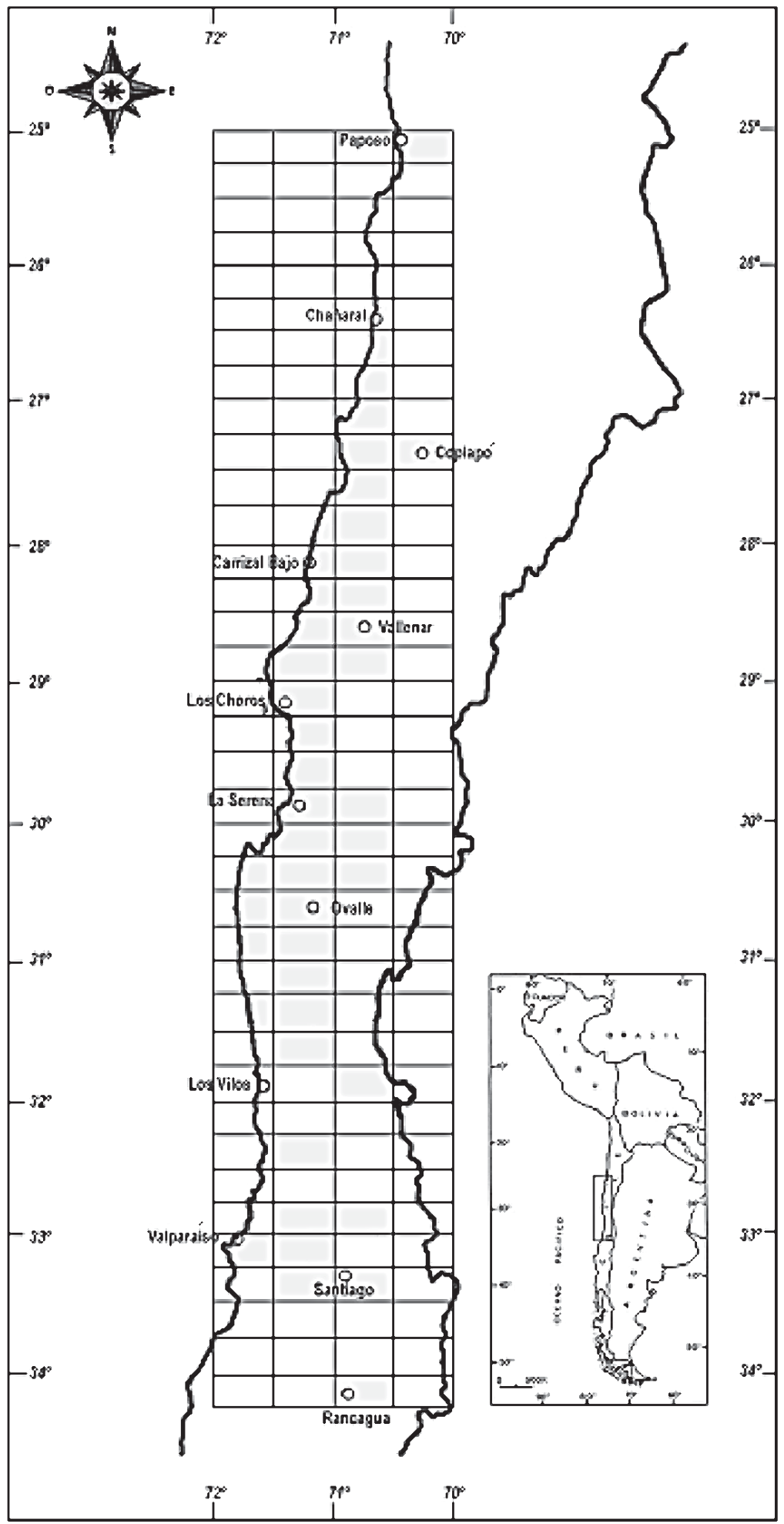

Fig. 1: Mapa de distribución de Gyriosomus y unidades operacionales de estudio (cuadrículas).

Distribution map of Gyriosomus and the operational units of study (grid cells). 


\section{TABLA 2}

Distribución de Gyriosomus en relación a formaciones vegetacionales descritas entre la II y VI Región de Chile. Abreviaturas: DCTa: desierto costero de Taltal; DCH: desierto costero del Huasco; DFLl: desierto florido de los llanos; DFS: desierto florido de las serranías; MEC: matorral estepario costero; MEB: matorral estepario boscoso; MEI: matorral estepario interior; MEA: matorral estepario arborescente; MAE: matorral andino esclerófilo; BEC: bosque esclerófilo costero; MECC: matorral espinoso de la cordillera de la costa

Gyriosomus distribution related with the vegetational formations described between II and VI Region of Chile. Abbreviations: DCTa: Taltal coastal desert; DCH: Huasco coastal desert; DFLl: plains florid deserts; DFS: hills florid desert; MEC: coastal steppe shrub; MEB: forest steppe shrub; MEI: interior steppe shrub; MEA: arborescent steppe shrub; MAE: sclerophyllous andean shrub; BEC: coastal sclerophyllous forest; MECC: coastal mountain range thorny shrub

\begin{tabular}{|c|c|c|c|c|c|c|c|c|c|c|c|c|}
\hline \multirow[b]{2}{*}{ ID } & \multirow[b]{2}{*}{ Especie } & \multicolumn{11}{|c|}{ Formaciones vegetacionales } \\
\hline & & DCTa & $\mathrm{DCH}$ & DFLl & DFS & MEC & MEB & MEI & MEA & MAE & BEC & MECC \\
\hline 1 & Gyriosomus hoppei & & & & & + & + & & & & & \\
\hline 2 & Gyriosomus impressus & & + & & & + & & & & & & \\
\hline 3 & Gyriosomus laevigatus & & & & & & & & & + & + & + \\
\hline 4 & Gyriosomus marmoratus & & & & + & & + & & & & & \\
\hline 5 & Gyriosomus luczotii & & & & & + & + & & & & & \\
\hline 6 & Gyriosomus bridgesi & & & & & + & + & + & & & & \\
\hline 7 & Gyriosomus elongatus & & & & & + & & & & & & \\
\hline 8 & Gyriosomus whitei & & + & & & + & & & & & & \\
\hline 9 & Gyriosomus planatus & & + & & & & & & & & & \\
\hline 10 & Gyriosomus parvus & & + & + & & & & & & & & \\
\hline 11 & Gyriosomus angustus & + & & & & & & & & & & \\
\hline 12 & Gyriosomus kingi & & + & & & & & & & & & \\
\hline 13 & Gyriosomus subrugatus & & + & + & & & & & & & & \\
\hline 14 & Gyriosomus curtisi & + & & & & & & & & & & \\
\hline 15 & Gyriosomus atacamensis & & + & & & & & & & & & \\
\hline 16 & Gyriosomus foveopunctatus & & & & & & & + & & & & \\
\hline 17 & Gyriosomus batesi & + & + & + & & & & & & & & \\
\hline 18 & Gyriosomus paulseni & & & & & & & + & & + & & \\
\hline 19 & Gyriosomus coriaceus & & & & & & & & + & & & \\
\hline 20 & Gyriosomus granocostatus & & & & & & & + & & & & \\
\hline 21 & Gyriosomus penicilliger & & & & & + & + & & & & & \\
\hline 22 & Gyriosomus planicollis & + & + & + & & & & & & & & \\
\hline 23 & Gyriosomus freyi & & & & & + & + & & + & & & \\
\hline 24 & Gyriosomus amabilis & & & & & + & & & & & & \\
\hline 25 & Gyriosomus leechi & & & + & + & + & + & + & & & & \\
\hline 26 & Gyriosomus reedi & & & & & + & + & + & & & & \\
\hline 27 & Gyriosomus barriai & & & & & & + & & & & & \\
\hline 28 & Gyriosomus gebieni & & + & & + & & & & & & & \\
\hline 29 & Gyriosomus lucens & & & & & & & & + & & & \\
\hline 30 & Gyriosomus pumilus & & & & & & & + & & + & & \\
\hline 31 & Gyriosomus penai & & & & & + & & & & & & \\
\hline 32 & Gyriosomus melcheri & & & & & & & & + & & & \\
\hline 33 & Gyriosomus modestus & & & & & & & & + & & & \\
\hline 34 & Gyriosomus kulzeri & & + & & & & & & & & & \\
\hline & Total & 4 & 11 & 5 & 3 & 12 & 9 & 7 & 5 & 3 & 1 & 1 \\
\hline
\end{tabular}

res se destaca el matorral estepario interior (MEI) y matorral estepario boscoso (MEB) con siete y nueve especies respectivamente. Las restantes formaciones presentan un menor número de especies.

\section{DISCUSIÓN}

Los patrones de distribución geográfica determinados para las especies de Gyriosomus, forman parte de las regiones mediterránea perárida, árida y semiárida sensu Di Castri (1968) y que representan zonas ecológicas y geomorfológicas particulares, definidas por formaciones vegetacionales asociadas a pulsos favorables de agua. Por ello, la marcada variabilidad interanual en los regímenes pluviométricos que existen en el norte de Chile y que constituye un recurso impredecible, sería uno de los factores que determinarían la presencia de Gyriosomus en formaciones vegetacionales específicas (Tabla 2) 
(Cepeda-Pizarro et al. 1996, Pizarro \& Jerez 2002 2 .

En el cladograma se observa las divergencias distribucionales del componente faunístico de Gyriosomus. El patrón de áreas anidadas (Fig. 2) permitió determinar dos áreas de en- demismo: una que corresponde a Paposo y que forma parte del desierto costero de Taltal (DCTa), y otra ubicada en planicies litorales que forman parte del desierto costero del Huasco (DCH). Sin embargo, la presencia de G. penai sinapomórfica para Los Choros, sec-

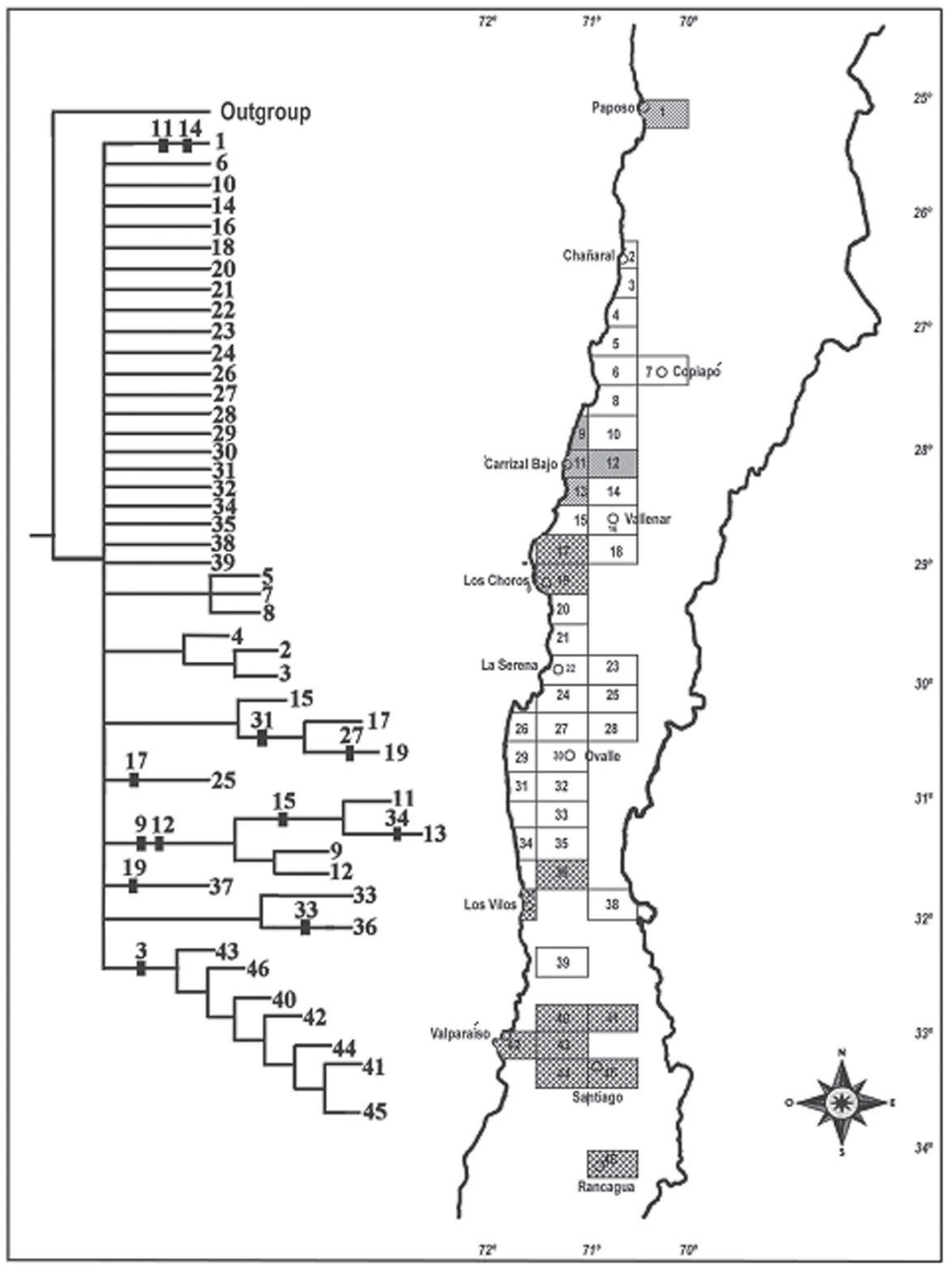

Fig. 2: Árbol de consenso estricto y áreas de endemismo para Gyriosomus: Paposo (Provincia de Antofagasta: II Región) y Carrizal Bajo (Provincia de Huasco: III Región).

Strict consensus tree and areas of endemism for Gyriosomus: Paposo (Province of Antofagasta: II Región) and Carrizal Bajo (Province of Huasco: III Región).

\footnotetext{
2 PIZARRO J \& V JEREZ (2002) Patrones de distribución geográfica del género Gyriosomus Guérin, 1834 (Coleoptera: Tenebrionidae). XXIV Congreso Nacional de Entomología. Santiago, Chile, Libro de Resúmenes: 25.
} 
tor contiguo a Carrizal Bajo, y G. laevigatus sinapomórfica para el matorral esclerófilo, estarían determinando otras posibles áreas de endemismo.

Estos resultados son congruentes en términos de concentración de especies endémicas con lo obtenido para la flora y fauna por otros autores. Paposo presenta 80 especies endémicas de vegetales (Cavieres et al. 2002) y 20 especies de Coleópteros (Jerez 2000). Hasta el momento no existe información sobre el sector de Carrizal Bajo en endemismo para la flora y fauna. Sin embargo el sector Choros Bajos presenta 15 especies endémicas de vegetales (Cavieres et al. 2001) y una especie de reptil, Liolaemus silvae (Ortiz 1989).

En términos de diversidad biológica, los ecosistemas del desierto costero del Huasco y matorral estepario costero, presentan la mayor diversidad específica de Gyriosomus con 11 y 12 especies respectivamente (Tabla 2), representando un continuo geográfico. Lo anterior podría representar zonas de "hotspots" para la diversidad de Gyriosomus y reforzaría lo señalado por Peña (1966a) e Irwin \& Schlinger (1986) en el sentido de considerar a la "Costa del Norte" como una zona ecológica diferente. Dentro de los ecosistemas interiores, se destaca el matorral estepario boscoso que presenta nueve especies, sin elementos endémicos y el matorral estepario interior con siete especies, de las cuales $G$. foveopunctatus es propia del sector.

Sin embargo, el bajo índice de consistencia mostrado por el cladograma de máxima-parsimonia, indica que varias especies de Gyriosomus presentan distribuciones amplias, especialmente a lo largo de la Depresión Intermedia. Este es el caso por ejemplo de G. leechi que se distribuye entre Vallenar $\left(28^{\circ} 34^{\prime} \mathrm{S} ; 70^{\circ} 45^{\prime} \mathrm{O}\right)$ y Punitaqui $\left(30^{\circ} 50^{\prime} \mathrm{S} ; 71^{\circ} 16^{\prime} \mathrm{O}\right)$ y $G$. luczotii con una distribución entre El Tofo $\left(28^{\circ} 38^{\prime} \mathrm{S}\right.$; $70^{\circ} 56^{\prime}$ O) y Socos $\left(30^{\circ} 44^{\prime}\right.$ S; 71 $31^{\prime}$ ' O). Estos rangos distribucionales latitudinales entre la cordillera de la Costa y la cordillera de los Andes que muestran las actuales especies de Gyriosomus junto a otros taxa de Tenebrionidae como ocurre con Entomoderes draco, ya se habrían establecido para sus ancestros probablemente desde principios del Eoceno, período en el cual se originó la región andinopatagónica o subantártica y Chile central (Roig-Juñent \& Flores 2001).

¿Cuáles serían las causas de endemismos en estos desiertos costeros?

Para Shmida (1985) el desierto costero del norte de Chile correspondería a un desierto anti- guo, anterior al levantamiento de la cordillera de la Costa, hecho sustentado por la presencia de una alta diversidad biótica con formas únicas y cuyo aislamiento dataría ya desde el Eoceno, periodo durante el cual el clima de la costa se habría vuelto más seco lo que generó las condiciones para la formación de estepas arbustivas xéricas. Roig-Juñent \& Flores (2001), señalan como componentes bióticos de estos ecosistemas a Gyriosomus (Tenebrionidae), Cnemalobus (Carabidae) y Elasmoderus (Tristiridae) y cactáceas como Echinopsis y Eulichnia que corresponderían a elementos paleo-endémicos adaptados a condiciones desérticas. En el caso de Gyriosomus estas adaptaciones están representadas por su apterismo y por el gran desarrollo de la cavidad subelitral.

Es probable que muestreos más exhaustivos permitan determinar otras áreas de endemismo o bien ampliar los rangos distribucionales de las especies. Al respecto, Jerez (2000) señala que la mayoría de la información disponible tanto en colecciones y literatura, corresponde a recolecciones efectuadas cerca de poblados o a la red vial, originando en conjunto una información local incompleta. Recolecciones en sectores de difícil acceso, como el límite costero sur del matorral estepario y la transecta costera entre Paposo y el Parque Nacional Pan de Azúcar, podrían incluir nuevos datos de distribución o establecer otras áreas de endemismo.

Finalmente los resultados obtenidos en este trabajo podrán ser interpretados a la luz de la biogeografía histórica, cuando se reconstruya la filogenia de Gyriosomus y se relacionen las divergencias evolutivas de los taxa con eventos vicariantes que pudieran haber afectado a su área de distribución.

\section{AGRADECIMIENTOS}

A Christian González y Jaime Solervicens del Instituto de Entomología UMCE; Ariel Camousseight de la sección Entomología del MNHN; Juan Carlos Ortiz y Luis Parra del Departamento de Zoología, Facultad de Ciencias Naturales y Oceanográficas de la Universidad de Concepción; Jorge Cepeda-Pizarro y Solange Vega del Laboratorio de Entomología Ecológica del Departamento de Biología de la Universidad de La Serena; Marcos Ferrú del Departamento de Ciencias del Mar, Universidad Arturo Prat, Iquique; Gustavo E. Flores del Instituto Argentino de Investigaciones de las Zonas Áridas IADIZACRICYT, Argentina y finalmente a Juan Enrique Barriga. Dos evaluadores anónimos y el editor asociado de la revista sugirieron valiosas modifi- 
caciones editoriales al manuscrito las que enriquecieron la versión final de este. Este trabajo fue financiado por el proyecto DIUC $\mathrm{N}^{\circ}$ 203.113.061-1.0 de la Dirección de Investigación, Universidad de Concepción.

\section{LITERATURA CITADA}

AGUILAR-AGUILAR R, R CONTRERAS-MEDINA \& G SALGADO-MALDONADO (2003) Parsimony analisis of endemicity (PAE) of Mexical hydrological basins on helminth parasites of freshwater fishes. Journal of Biogeography 30: 1.861-1.872.

AMAT-GARCÍA G \& DR MIRANDA-ESQUIVEL (1996) Insectos, biodiversidad, conservación: ¿Cómo monitorear insectos en Colombia? En: Andrade GMG, G Amat-García \& F Fernández (eds) Insectos de Colombia: 37-64. Academia Colombiana de Ciencias Exactas, Físicas y Naturales. Santa Fe de Bogotá, Colombia.

CABRERA A \& A WILLINK (1973) Biogeografía de América Latina. Monografías de la Organización de los Estados Americanos, Serie Biología. 122 pp.

CAMUS P (2001) Biogeografía marina de Chile continental. Revista Chilena de Historia Natural 74: 587-617.

CAVIERES LA, M MIHOC, A MARTICORENA, C MARTICORENA, O MATTHEI \& FA SQUEO (2001) Determinación de áreas prioritarias de conservación: análisis de parsimonia de endemismos (PAE) en la flora de la IV Región de Coquimbo. En: Squeo FA, G Arancio \& JR Gutiérrez (eds) Libro rojo de la flora nativa y de los sitios prioritarios para su conservación: Región de Coquimbo: 159170. Ediciones Universidad de La Serena, La Serena, Chile.

CAVIERES LA, MTK ARROYO, P POSADAS, C MARTICORENA, O MATTHEI, R RODRÍGUEZ, FA SQUEO \& G ARANCIO (2002) Identification of priority areas for conservation in an arid zone: application of parsimony analysis of endemicity in the vascular flora of the Antofagasta region, northern Chile. Biodiversity and Conservation 11: 1.303-1.311.

CEPEDA-PIZARRO JG (1989) Actividad temporal de tenebriónidos epigeos (Coleoptera) y su relación con la vegetación arbustiva en un ecosistema árido de Chile. Revista Chilena de Historia Natural 62: 115-125.

CEPEDA-PIZARRO JG, H VÁSQUEZ, H VEAS \& G COLON (1996) Relaciones entre tamaño corporal y biomasa de Tenebrionidae (Coleoptera) de la estepa costera del margen meridional del desierto chileno. Revista Chilena de Historia Natural 69: 67-76.

Di CASTRI F (1968) Esquisse écologique du Chili. En: Delamare CL \& E Rapoport (eds) Biologie de 1'Amérique australe: 7-52. Editions Centre National de la Recherche Scientifique, Paris, France.

DONATO M, P POSADAS, DR MIRANDA-ESQUIVEL, EO JAUREGUIZAR \& G CLADERA (2003) Historical biogeography of the Andean region: evidence from Listroderina (Coleoptera: Curculionidae: Rhytirrhinini) in the context of the South American geobiotic scenario. Biological Journal of the Linnean Society 80: 339-352.

EGUIARTE LE, J LARSON-GUERRA, J NÚÑEZ-FARFÁN, A MARTÍNEZ-PALACIOS, K SANTOS DEL PRADO \& HT ARITA (1999) Diversidad filogenética y conservación: ejemplos a diferentes escalas. Revista Chilena de Historia Natural 72: 475-492.
ESCALANTE T \& JJ MORRONE (2003) ¿Para qué sirve el análisis de parsimonia de endemismos? En: Morrone JJ \& J Llorente (eds) Una perspectiva latinoamericana de la biogeografía: 167-172. Las Prensas de Ciencias, UNAM, México, Distrito Federal.

ESCALANTE T, D ESPINOSA \& JJ MORRONE (2003) Using parsimony analysis of endemicity to analyze the distribution of Mexican land mammals. Southwestern Naturalist 48: 563-578.

ESPADAS-MANRIQUE C, R DURÁN \& J ARGÁEZ (2003) Phytogeographic analysis of taxa endemic to the Yucatán Peninsula using geographic information systems, the domain heuristic method and parsimony analysis of endemicity. Diversity and Distributions 9: 313-330.

FAIRMAIRE L (1876) Révision des Coléoptères du Chili. Famille des Tenebrionidae, Tribu des Nyctélites. Annales de la Société Entomologique de France 5: 1ère partie: 143-170; 2ème partie: 341-383.

FATTORINI S (2002) Biogeography of the tenebrionid beetles (Coleoptera, Tenebrionidae) on the Aegean Islands (Greece). Journal of Biogeography 29: 49-67.

FLORES GE (1997) Revisión de la tribu Nycteliini (Coleoptera: Tenebrionidae). Revista de la Sociedad Entomológica Argentina 56: 1-19.

FLORES GE (2000) Cladistic analysis of the Neotropical tribe Nycteliini (Coleoptera: Tenebrionidae). Journal of the New York Entomological Society 108: 13-25.

GAJARDO R (1994) La Vegetación Natural de Chile: clasificación y distribución geográfica. Editorial Universitaria, Santiago, Chile. 165 pp.

GARCÍA-BARROS E, P GURREA, MJ LUCIÁÑEZ, JM CANO, ML MUNGUIRA, JC MORENO, H SANZ \& JC SIMON (2002) Parsimony analysis of endemicity and its application to animal and plant geographical distributions in the Ibero-Balearic region (western Mediterranean). Journal of Biogeography 29: 109-124.

GEBIEN H (1910). Tenebrionidae. I: 1-166; II: 167-354. Coleopterorum catalogus. Berlin, Germany. 18 pts. $15,22$.

GOLOBOFF P (1997) NONA versión 2.0 Published by the autor.

IRWIN M \& E SCHLINGER (1986) A gazetteer for the 1966-1967, University of California-Universidad de Chile arthropod expedition to Chile and parts of Argentina. Occasional Papers of the California Academy of Science (USA) 144: 1-11.

JEREZ V (2000) Diversidad y patrones de distribución geográfica de insectos coleópteros en ecosistemas desérticos de la región de Antofagasta, Chile. Revista Chilena de Historia Natural 73: 79-92.

KASZAB Z (1969) The scientific result of the Hungarian soil zoological expeditions to South America Tenebrioniden aus Chile (Coleoptera). Opus Zoological Budapest 9: 291-337.

KULZER H (1954) Neunter Beitrag zur Kenntnis der Tenebrioniden (Col.) Eine Studie über die Tribus Nycteliini. Entomologische Arbeiten aus dem Museum George Frey 5: 145-267.

KULZER H (1959) Neue Tenebrioniden aus Südamerika (Col.) 18 Beitrag zur Kenntnis der Tenebrioniden. I Die Gattung Gyriosomus Guérin. (Nycteliini). Entomologische Arbeiten aus dem Museum George Frey 10: 523-547.

KUSCHEL G (1964) Problems concerning an austral region. En: Gressit JL, CH Lindroth, FR Forsberg, A Fleming \& EG Turbott (eds) Pacific basin biogeography: 443-449. Bishop Museum Press, Honolulu, Hawai, USA. 
KUSCHEL G (1969) Biogeography and ecology of South American Coleoptera. En: Fittkau J, H Kinge, GH Schawake \& H Sioli (eds) Biogeography and ecology in South America 709-722. Dr W Junk Publishers, The Hague, The Netherlands.

MORRONE JJ (1994) On the identification of areas of endemism. Systematic Biology 43: 438-441.

MORRONE JJ, S ROIG-JUÑENT \& JV CRISCI (1994) Cladistic biogeography of terrestrial Sub-Antartic Beetles (Insecta: Coleoptera) from South America. National Geographic Research \& Exploration 10: 104-115.

MORRONE JJ \& JV CRISCI (1995) Historical biogeography: introduction to methods. Annual Review of Ecology and Systematics 26: 373-401.

MORRONE JJ (1996) The biogeographical Andean subregion: a proposal exemplified by Arthropod taxa (Arachnida, Crustacea and Hexapoda). Neotropica 42: $103-114$

MORRONE JJ, L KATINAS \& JV CRISCI (1997) A cladistic biogeographic analysis of Central Chile. Journal of Comparative Biology 2: 25-42.

MORRONE JJ \& T ESCALANTE (2002) Parsimony Analysis of Endemicity (PAE) of Mexican terrestrial mammals at different area units: when size matters. Journal of Biogeography 29: 1-10.

MORRONE JJ, S ROIG-JUNENT \& G FLORES (2002) Delimitation of biogeographic districts in central Patagonia (South America), based on beetle distributional patterns (Coleoptera: Carabidae and Tenebrionidae). Revista del Museo Argentino de Ciencias Naturales 4: 1-6.

MYERS N, RA MITTERMEIER, CG MITTERMEIER, GAB DA FONSECA \& J KENT (2000) Biodiversity hotspots for conservation priorities. Nature 403 : 853-858.

NIXON KC (2000) Winclada. Published by the autor.

ORMAZÁBAL CS (1993) The conservation of biodiversity in Chile. Revista Chilena de Historia Natural 66: 383-402.

ORTIZ JC (1989) Descripción de Liolaemus silvae sp. nov. (Sauria, Iguanidae) du "norte chico" du Chili. Bulletin du Muséum National d'Histoire Naturelle de Paris. 4ème série, 11 Section A 1: 247-252.

PASKOFF R (1993) Geomorfología de Chile Semiárido. Departamento de Publicaciones Universidad de La Serena, La Serena, Chile. 321 pp.

PEÑA LE (1959) Las vaquitas del desierto. Noticiero Mensual del Museo Nacional de Historia Natural (Chile) 37: 1-2.

PEÑA LE (1966a) Ensayo preliminar para dividir Chile en regiones entomofaunísticas, basadas especialmente en la familia Tenebrionidae (Col). Apartado de la Revista Universitaria 51: 210-220.

PEÑA LE (1966b) Catálogo de los Tenebrionidae (Coleoptera) de Chile. Entomologische Arbeiten aus dem Museum George Frey 17: 397-453.

PHILIPPI F (1887) Catálogo de los Coleópteros de Chile. Anales de la Universidad de Chile 71: 1-190.

POSADAS P (1996) Distributional patterns of vascular plants in Tierra del Fuego: a study applying parsimony analysis of endemicity (PAE). Biogeographica 72: 161-177.

POSADAS P \& D MIRANDA-ESQUIVEL (1999) El PAE (Parsimony analysis of endemicity) como una herramienta en la evaluación de la biodiversidad. Revista Chilena de Historia Natural 72: 539-546.

ROIG-JUÑENT S \& GE FLORES (2001) Historia geográfica de las áreas áridas de América del Sur Austral. En: Llorente Busquets J \& JJ Morrone (eds) Introducción a la biogeografía en Latinoamérica: teorías, conceptos, métodos y aplicaciones: 257-266. Las prensas de Ciencias, Facultad de Ciencias, UNAM, México, Distrito Federal.

ROIG-JUÑENT S, GE FLORES \& C MATTONI (2003) Consideraciones biogeográficas de la Precordillera (Argentina), con base en artrópodos epígeos. En: Morrone JJ \& J Llorente-Bousquets (eds) Una perspectiva latinoamericana de la biogeografía: 275288. Las Prensas de Ciencias, Facultad de Ciencias, UNAM, México, Distrito Federal.

SAIZ F, J SOLERVICENS \& P OJEDA (1989) Coleópteros del Parque Nacional La Campana y Chile Central. Ediciones Universitarias de Valparaíso, Valparaíso, Chile. 124 pp.

SHMIDA A (1985) Biogeography of the desert flora. En: Evenari AM, I Noy-Meir \& DV Goodall (eds) Hot deserts and arid shrublands: 23-77. Elsevier, New York, New York, USA.

SQUEO FA, LA CAVIERES, G ARANCIO, JE NOVOA, O MATTHEI, C MARTICORENA, R RODRÍGUEZ, MTK ARROYO \& M MUÑOZ (1998) Biodiversidad de la flora vascular en la Región de Antofagasta, Chile. Revista Chilena de Historia Natural 71: 571-591.

SZUMIK C, F CUEZZO, P GOLOBOFF \& A CHALUP (2002) An optimality criterion to determine areas of endemism. Systematic Biology 51: 806-816.

WATERHOUSE GR (1844) Contribution to the entomology of the southern portions of South America. Annals and Magazine of Natural History 13: 41-55. 